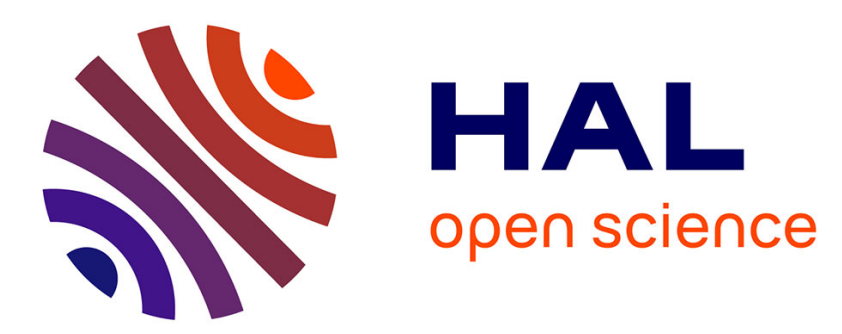

\title{
Singularity of cable-driven parallel robot with sagging cables: preliminary investigation \\ Jean-Pierre Merlet
}

\section{To cite this version:}

Jean-Pierre Merlet. Singularity of cable-driven parallel robot with sagging cables: preliminary investigation. ICRA 2019 - IEEE International Conference on Robotics and Automation, May 2019, Montreal, Canada. hal-02426398

\section{HAL Id: hal-02426398 https://hal.inria.fr/hal-02426398}

Submitted on 2 Jan 2020

HAL is a multi-disciplinary open access archive for the deposit and dissemination of scientific research documents, whether they are published or not. The documents may come from teaching and research institutions in France or abroad, or from public or private research centers.
L'archive ouverte pluridisciplinaire HAL, est destinée au dépôt et à la diffusion de documents scientifiques de niveau recherche, publiés ou non, émanant des établissements d'enseignement et de recherche français ou étrangers, des laboratoires publics ou privés. 


\title{
Singularity of cable-driven parallel robot with sagging cables: preliminary investigation
}

\author{
J-P. Merlet ${ }^{1}$
}

\begin{abstract}
This paper addresses for the first time the singularity analysis of cable-driven parallel robot (CDPR) with sagging cables using the Irvine model. We present the mathematical framework of singularity analysis of CDPR using this cable model. We then show that, besides a cable model representation singularity, both the inverse and forward kinematics (IK and FK) have a singularity type, called parallel robot singularity, which correspond to the singularity of an equivalent parallel robot with rigid legs. We then show that both the IK and FK have also full singularities, that are not parallel robot singularity and are obtained when two of the IK or FK solution branches intersect. IK singularity will usually lie on the border of the CDPR workspace. We then exhibit an algorithm that allow one to prove that a singularity exist in the neighborhood of a given pose and to estimate its location with an arbitrary accuracy. Examples are provided for parallel robot, IK and FK singularities. However we have not been able to determine examples of combined singularity where both the IK and FK are singular (besides parallel robot singularity).

Index terms: parallel robot cable-driven parallel robot, singularity
\end{abstract}

\section{INTRODUCTION}

We consider a variant of parallel robots, namely cabledriven parallel robots (CDPR), in which the rigid legs of parallel robots are substituted by coilable/uncoilable cables. Cables are coiled on a winch whose cable output is a fixed point $A$, while the cable extremity $B$ is attached to the endeffector. at point $B$ (figure 1). For CDPR any kinematic problem involves also cable tensions as cables can exert only a pulling action so that the name kineto-static is often used. Consequently kinematic modeling of CDPR is highly dependent upon the statics modeling of cable. In a vast majority of works cables are assumed to be ideal i.e. the cable shape is the straight line between $A$ and $B$ and its length $L_{0}$ is independent from the cable tension $\tau$, figure 1 . Some works use an elastic model in which the shape is the same than for ideal cable but the cable length $\rho$ is linearly related to the cable tension by $\rho=L_{0}+\tau / k$ where $L_{0}$ is the length at rest of the cable and $k$ the stiffness of the cable.

In both cases a cable has an influence on the end-effector iff $\tau>0$, otherwise the cable is slack and this will occur if $L_{0}>\|\mathbf{A B}\|$. For a given pose $\mathbf{X}$ of the end-effector there is a linear relationship between the external wrench $\mathcal{F}$ (without loss of generality we will assume that the endeffector is submitted only to its own weight) that is acting on the end-effector and the cable tensions:

$$
\mathcal{F}=\mathbf{J}^{-\mathbf{T}}(\mathbf{X}) \tau
$$

${ }^{1}$ HEPHAISTOS project, Université Côte d'Azur, Inria, France Jean-Pierre.Merlet@inria.fr

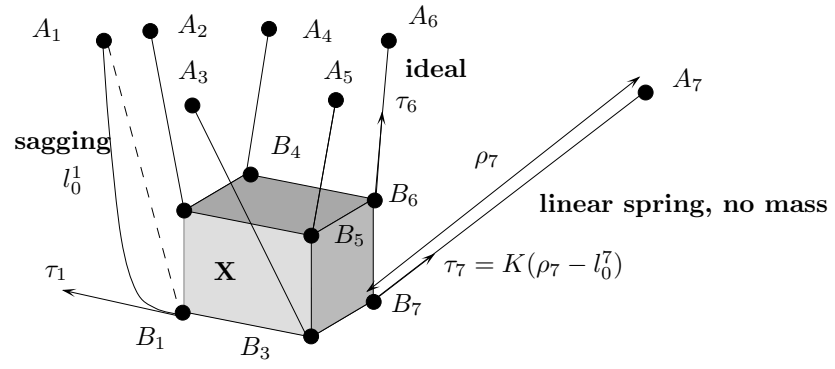

Fig. 1. A CDPR and various cable model

where $\mathbf{J}^{-\mathbf{T}}$ is the transpose of the inverse kinematic jacobian, that is pose dependent. The $i-t h$ column of this matrix is $\left(\mathbf{A}_{\mathbf{i}} \mathbf{B}_{\mathbf{i}} /\left\|\mathbf{A}_{\mathbf{i}} \mathbf{B}_{\mathbf{i}}\right\|, \mathbf{C B}_{\mathbf{i}} \times \mathbf{A}_{\mathbf{i}} \mathbf{B}_{\mathbf{i}} /\left\|\mathbf{A}_{\mathbf{i}} \mathbf{B}_{\mathbf{i}}\right\|\right)^{T}$, where $C$ is the center of mass of the end-effector. Note that $\mathbf{J}^{-\mathbf{T}}$ is established by using only non slack cables. A singularity will occur if $\mathbf{J}^{-\mathbf{T}}$ is not full rank and consequently a CDPR has the same singularity than a parallel robot with rigid legs [1] and there is no other singularity (the force-closure singularity mentioned in [2], [3] is just a border curve that is not related a singularity of the equations). The consequence of such a singularity (loss of control, large tensions) and the singularity location has been studied in depth for parallel robot [4], [5], [6], [7].

However for large CDPR the assumption of ideal or elastic cable will not hold because the cable own mass cannot be neglected and we have to assume that the cables are sagging. Very few studies consider cable models that are more realistic than the ideal cable [8], [9], [10], [11], although they will radically change the mathematical structure of CDPR models. To the best of the author knowledge singularity analysis of CDPR with sagging cables has never been addressed and this paper presents a preliminary investigation of this topic.

\section{CABle MOdel AND Kinematics}

Regarding cable there are models developed from civil and maritime engineering that are more realistic than the ideal cable model: some of them take into account torsion, out-of-plane motion [12], [13], the multi-strand nature of the cable [14] or are specific to synthetic rope [14], [15], [16], [17], [18]. In this paper we will use the Irvine sagging cable model that is valid for elastic and deformable cable with mass [19] and that has been shown to be in very good agreement with experimental results [20]. Note that as mentioned by the reviewers attributing this model to Irvine may possibly be not exact. This model assumes that the 
cable lies in a vertical plane and is a 2D model (figure 2).

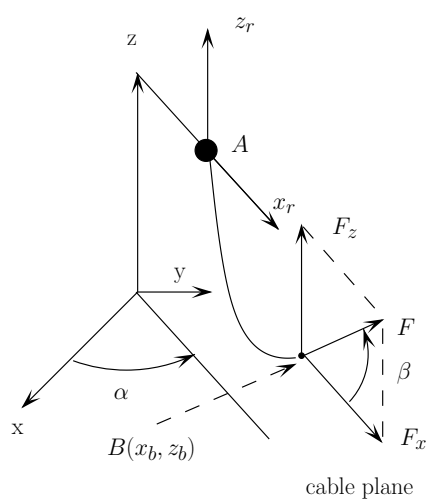

Fig. 2. Notation for a sagging cable

In this plane a frame is defined with origin the upper attachment point of the cable $A(0,0)$ and horizontal and vertical axis $x_{r}, z_{r}$. The cable lower attachment point $B$ has as coordinates $\left(x^{r} \geq 0, z^{r}<0\right)$. Note that we assume here that $B$ is always below $A$ (ie. we are considering a suspended CDPR) but this restriction is not essential. Vertical and horizontal forces $F_{z}, F_{x}>0$ are exerted on the cable at point $B_{i}$. For a cable with length at rest $L_{0}$ the coordinates of $B$ are given by [19]:

$$
\begin{aligned}
& x^{r}=F_{x}\left(\frac{L_{0}}{E A_{0}}+\frac{\sinh ^{-1}\left(F_{z}\right)-\sinh ^{-1}\left(F_{z}-\mu g L_{0} / F_{x}\right)}{\mu g}\right) \\
& z^{r}=\frac{F_{z}}{E A_{0}}-\frac{\mu g L_{0}^{2}}{2}+\frac{\sqrt{F_{x}^{2}+F_{z}^{2}}-\sqrt{F_{x}^{2}+\left(F_{z}-\mu g L_{0}\right)^{2}}}{\mu g}(2)
\end{aligned}
$$

where $E$ is the Young modulus, $A_{0}$ is the cable cross-section area, and $\mu$ is the cable linear density.

We consider a spatial CDPR with $n \geq 3$ cables and define a reference frame $(O, \mathbf{x}, \mathbf{y}, \mathbf{z})$ with a vertical $\mathbf{z}$ axis. For cable $i$ a rotation around the $\mathbf{z}$ axis of angle $\alpha_{i}$ allows one to transform the coordinates $\left(x_{i}, y_{i}, z_{i}\right)$ of $A_{i} B_{i}$ in the reference frame to its coordinates $x_{i}^{r}, z_{i}^{r}$ in the cable plane (figure 2). We have $x_{i}^{r}=x_{i} \cos \alpha_{i}+y_{i} \sin \alpha_{i}, z_{i}^{r}=z_{i}$ and the angle $\alpha_{i}$ must satisfy:

$$
-x_{i} \sin \left(\alpha_{i}\right)+y_{i} \cos \left(\alpha_{i}\right)=0 \quad x_{i}^{r}>0
$$

If $\left(F_{x_{i}}>0, F_{z_{i}}\right)$ are the components of the force that is applied by the platform on the cable at $B_{i}$ in its plane, then the force exerted by the cable on the platform in the reference frame is $\mathbf{F}_{\mathbf{a}}^{\mathbf{i}}=\left(-F_{x_{i}} \cos \left(\alpha_{i}\right),-F_{x_{i}} \sin \left(\alpha_{i}\right),-F_{z_{i}}\right)$. We assume that the platform is subjected only to gravity which exert a vertical force $\mathcal{F}$ and no torque around the platform center of mass $C$. Hence the mechanical equilibrium imposes

$$
\sum_{j=1}^{j=n} \mathbf{F}_{\mathbf{a}}^{\mathbf{j}}+\mathcal{F}=\mathbf{0} \quad \sum_{j=1}^{j=n} \mathbf{C B}_{\mathbf{i}} \times \mathbf{F}_{\mathbf{a}}^{\mathbf{j}}=\mathbf{0}
$$

We may also provide an alternate form for the kinematic equations. Let $\mathbf{n}_{\mathbf{i}}$ be the tangent vector to the cable at $B_{i}$ and let $\tau_{i}=\sqrt{F_{x_{i}}^{2}+F_{z_{i}}^{2}}$. Using the notation of figure 2 we have $\mathbf{n}_{\mathbf{i}}=\left(\cos \beta_{i} \cos \alpha_{i}, \cos \beta_{i} \sin \alpha_{i}, \sin \beta_{i}\right)^{T}$ so that
$F_{x_{i}}=\tau_{i} \cos \beta_{i}, F_{z_{i}}=\tau_{i} \sin \beta_{i}$ and the Irvine equations may be written as function of $\tau_{i}, \alpha_{i}, \beta_{i}, L_{0}^{i}$. The force exerted by cable $i$ on the platform is therefore $\tau_{i} \mathbf{n}_{\mathbf{i}}$ and if $\tau$ denotes the $\tau_{i}$ s array, we have

$$
\mathbf{J} \tau=(\mathcal{F}, \mathbf{0})^{T}
$$

where a column of matrix $\mathbf{J}$ is $\left(\mathbf{n}_{\mathbf{i}}, \mathbf{C B}_{\mathbf{i}} \times \mathbf{n}_{\mathbf{i}}\right)^{T}$. Note that this matrix is similar to the matrix that will be obtained for a parallel robot with rigid legs whose $A_{i}$ will be located on the line going through $B_{i}$ with unit vector $\mathbf{n}_{\mathbf{i}}$. This robot will be called the parallel equivalent robot of the CDPR.

Kinematic analysis will be based on the $2 n$ equations (2) (denoted $E_{I}$ ), the $n$ equations (3) (denoted $E_{\alpha}$ ) and the 6 equations (4) (denoted $E_{s}$ ) for a total of $3 n+6$ constraints that involves the $4 n$ variables $F_{x_{i}}, F_{z_{i}}, L_{0}^{i}, \alpha_{i}$ and the 6 pose $\mathbf{X}$ parameters. An equivalent system in $\tau_{i}, L_{0}^{i}, \alpha_{i}, \beta_{i} \mathbf{X}$ is obtained using (5) as $E_{s}$ and $F_{x_{i}}=\tau_{i} \cos \beta_{i}, F_{z_{i}}=\tau_{i} \sin \beta_{i}$. We define the actuation lengths array $\Theta_{\mathbf{a}}=\left\{L_{0}^{i}\right\}$ of dimension $n$, the auxiliary variable array $\Theta_{\mathbf{p}}=\left\{F_{x_{i}}, F_{z_{i}}, \alpha_{i}\right\}$ (or $\left\{\tau_{i}, \beta_{i}, \alpha_{i}\right\}$ ) of dimension $3 n$, while $\mathbf{W}$ will be the twist of the platform. The array $\Theta_{\mathbf{p}}$ may be split in $3 n$ dimensional arrays $\boldsymbol{\Theta}_{\mathbf{p}_{1}}=\left\{F_{x_{i}}\right\}$ (or $\left\{\tau_{i}\right\}$ ), $\boldsymbol{\Theta}_{\mathbf{p}_{2}}=\left\{F_{z_{i}}\right\}$ (or $\left\{\beta_{i}\right\}$ ) and $\boldsymbol{\Theta}_{\mathbf{p}_{3}}=\left\{\alpha_{i}\right\}$. Differentiating the constraints and rearranging them in order to use the relationship between $\dot{\mathbf{X}}$ and $\mathbf{W}$ allows one to establish the kinetic relationship:

$$
\mathrm{A} \dot{\Theta}_{\mathrm{a}}+\mathrm{BW}+\mathrm{C} \dot{\Theta}_{\mathrm{p}}=\mathbf{0}
$$

where $\mathrm{A}$ is a $(3 n+6) \times n$ matrix, $\mathrm{B}$ is $(3 n+6) \times 6$ and $\mathrm{C}$ is $(3 n+6) \times 3 n$. These matrices may be written as:

$\mathrm{A}=\left(\begin{array}{c}\mathrm{A}^{\prime} \\ \mathbf{0}_{n+6 \times n}\end{array}\right) \quad \mathrm{B}=\left(\begin{array}{c}\mathrm{B}_{1} \\ \mathrm{~B}_{2} \\ \mathbf{0}_{6 \times 6}\end{array}\right) \quad \mathrm{C}=\left(\begin{array}{ccc}\mathrm{C}_{1} & \mathrm{C}_{2} & \mathrm{C}_{3} \\ \mathbf{0}_{n \times 2 n} & & \mathrm{C}_{1}^{\alpha} \\ \mathrm{C}_{1}^{s} & \mathrm{C}_{2}^{s} & \mathrm{C}_{3}^{s}\end{array}\right)$

where $\mathrm{A}^{\prime}=\partial E_{I} / \partial L_{0}$ is $2 n \times n, \mathrm{~B}_{1}=\partial E_{I} / \partial \mathbf{W}$ is $2 n \times 6$, $\mathrm{B}_{2}=\partial E_{\alpha} / \partial \mathbf{W}$ is $n \times 6$.

Matrix $\mathrm{C}_{1}=\partial E_{I} / \partial \boldsymbol{\Theta}_{\mathbf{p}_{1}}$ is $2 n \times n, \mathrm{C}_{2}=\partial E_{I} / \partial \boldsymbol{\Theta}_{\mathbf{p}_{2}}$ is $2 n \times n$, $\mathrm{C}_{3}=\partial E_{I} / \partial \boldsymbol{\Theta}_{\mathbf{p}_{3}}$ is $2 n \times n$ and $\mathrm{C}_{1}^{\alpha}=\partial E_{\alpha} / \partial \alpha$ is $n \times n$. We have also $C_{i}^{s}=\partial E_{s} / \partial \Theta_{\mathbf{p}_{i}}$, all these matrices being of dimension $6 \times n$. Note that if $\boldsymbol{\Theta}_{\mathbf{p}}=\left\{\tau_{i}, \beta_{i}, \alpha_{i}\right\}$, then $\mathrm{C}_{1}^{s}=\mathbf{J}$ is the matrix constituted of the Plücker vectors of the lines associated to the cable tangent at $B_{i}$, while $\mathrm{C}_{2}^{s}$ and $\mathrm{C}_{3}^{S}$ are constituted of the non normalized Plücker vectors of lines going through $B_{i}$, that are perpendicular to the cable tangents. Furthermore a line of $\mathrm{C}_{2}^{s}$ is perpendicular to its corresponding line in $\mathrm{C}_{3}^{s}$. It must also be noted that the rank of $\mathrm{C}_{3}^{s}$ is always lover than 6 as $\left|\mathrm{C}_{3}^{s}\right|=0$. If $\boldsymbol{\Theta}_{\mathbf{p}}=\left\{F_{x_{i}}, F_{z_{i}},, \alpha_{i}\right\}$ let $\mathbf{V}$ denotes the matrix $\left(\begin{array}{ll}\mathrm{C}_{1}^{s} & \mathrm{C}_{2}^{s}\end{array}\right)$ and $\mathbf{U}$ the $2 n \times n$ matrix whose elements of the $i$-th column are 0 except for $\mathbf{U}[2 i-1, i]=\cos \beta_{i}$ and $\mathbf{U}[2 i, i]=\sin \beta_{i}$. The rank of $\mathbf{U}$ is always $n$ and we have $\mathbf{V U}=\mathbf{J}$ so that the rank of $\mathbf{V}$ is equal to the rank of $\mathbf{J}$.

In this paper we will first consider the singularity for the inverse kinematics (IK): the pose $\mathbf{X}$ is known (and consequently the angles $\alpha_{i}$ can be calculated), while the $F_{x_{i}}, F_{z_{i}}, L_{0}^{i}$ (or equivalently $\tau_{i}, \beta_{i}, L_{0}^{i}$ ) are unknown. We have $2 n+6$ equations $(2,4)$ for $3 n$ unknowns. 
We will then consider the forward kinematics (FK): the lengths $L_{0}^{i}$ are known, while $\mathbf{X}, F_{x_{i}}, F_{z_{i}}, \alpha_{i}$ are unknown. We have $3 n+6$ equations (2,3 4$)$ for $3 n+6$ unknowns.

\section{INVERSE KINEMATICS SINGULARITY}

As seen in the previous section the IK equation system may be written as $\mathbf{F}_{\mathbf{I K}}\left(L_{0}^{i}, F_{x_{i}}, F_{z_{i}}\right)=0$ (or $\mathbf{F}_{\mathbf{I K}}\left(L_{0}^{i}, \tau_{i}, \beta_{i}\right)=0$ ) as $\alpha_{i}$ has a fixed value. A singularity will be obtained when the jacobian $\mathbf{J}_{\mathbf{I K}}$ of this system (which is of dimension $(2 n+$ $6) \times 3 n$ ) is not full rank. If $n<6$ the IK is overconstrained, unless we let $6-n$ pose parameters be free in which case the system becomes square. If $n=6$ the IK is a square system, while if $n>6$ the system is underconstrained. We already know that for $n=6$ the IK may have multiple solutions [21]. Using equation (6) we get

$$
\mathbf{J}_{\mathbf{I K}}=\left(\begin{array}{ccc}
\mathrm{A}^{\prime} & \mathrm{C}_{1} & \mathrm{C}_{2} \\
\mathbf{0}_{6 \times n} & \mathrm{C}_{1}^{s} & \mathrm{C}_{2}^{s}
\end{array}\right)
$$

The matrix $\mathbf{J}_{\mathbf{I K}}$ may be singular in three cases:

- IK Irvine singularity: when $F_{x_{i}}=0$ we have a singularity of the Irvine equations. This is however a removable singularity [22] as $F_{x} x^{r} \rightarrow 0$ when $F_{x} \rightarrow 0$. We can redefine $x^{r}$ in such a way that the Irvine equations will be regular: here we will define $x^{r}\left(F_{x}=0\right)=0$ for that purpose as 0 is the limit of $x^{r}$ when $F_{x}$ goes to 0 .

- IK parallel robot singularity: when $\mathbf{J}$ is singular which correspond to a singularity of the parallel equivalent robot. Here the mechanical equilibrium equation system is not full rank and we may have an infinitesimal motion of the platform without any change in the $L_{0}$

- IK full singularity: if $\mathbf{J}$ is not singular and two (or more) solution branches of the IK meet. In that case there are directions for the platform twist that have no solution in terms of actuation velocities. Usually these poses lie on the border of the CDPR workspace.

\section{A. Examples}

Our purpose is to show that IK parallel robot and full singularity may exist.

1) IK parallel robot singularity: We consider a 6-cables CDPR where the $A_{i}, B_{i}$ are equally distributed on circles of different radii with centers $C_{A}, C_{B}$ and we assume that the circle with the $A_{i}$ is horizontal. Then we place the platform in a pose so that the platform circle is horizontal and the centers $C_{A}, C_{B}$ lie on the same vertical line $V$. In that configuration because of the symmetry an IK solution is obtained with the same tension in each cable and the tangents of the cables at $B_{i}$ all intersect the same point on $V$, leading to a singularity of $\mathbf{J}$ [23]. The $\mathbf{J}$ matrix is $6 \times 6$ buts is rank is only 3 leading to a rank of 15 for the matrix $\mathbf{J}_{\mathbf{I K}}$ instead of 18: this is a typical example of IK parallel robot singularity.

2) IK full singularity: We consider as example our large scale robot MARIONET-CRANE [24], one of the largest CDPR ever deployed. This robot is a suspended CDPR with 6 cables, whose $A_{i}, B_{i}$ coordinates are given in table I (in cm for clarity but meters are used for the calculation). The IK jacobian $\mathbf{J}_{\mathbf{I K}}$ of this CDPR is a square matrix of dimension

\begin{tabular}{|c|c|c|c|c|c|c|c|}
\hline$x_{A}$ & \multicolumn{2}{|c|}{$y_{A}$} & $z_{A}$ & $x$ & \multicolumn{2}{|c|}{$y_{A}$} & $z_{A}$ \\
\hline-325.9 & \multicolumn{2}{|c|}{-47.5} & 82.6 & 942 & \multicolumn{2}{|c|}{-348.2} & 1155.5 \\
\hline 953.8 & \multicolumn{2}{|c|}{379.7} & 153.3 & 557 & \multicolumn{2}{|c|}{2041.4} & 870.4 \\
\hline-250.5 & \multicolumn{2}{|c|}{1681.0} & 64.9 & -33 & \multicolumn{2}{|c|}{942.1} & 878.8 \\
\hline & $x_{B}$ & $y_{B}$ & $z_{B}$ & $x_{B}$ & $y_{B}$ & $z_{B}$ & \\
\hline & -10 & -93 & -3 & 10 & -93 & -3 & \\
\hline & 27 & 50 & -7 & 27 & 50 & -7 & \\
\hline & -27 & 50 & -7 & -27 & 50 & -7 & \\
\hline
\end{tabular}

TABLE I

Coordinates of the $A_{i}$ AND $B_{i}$ POINTS on the BASE AND ON THE PLATFORM (UNIT: CM, ORDERED BY ROW))

$18 \times 18$. A pose of this robot is defined by the coordinates $x_{g}, y_{g}, z_{g}$ of the platform center of mass and by the rotation matrix $\mathbf{R}$ between the mobile and reference frames. To find an IK full singularity we have considered the pose with coordinates in meters $x_{g}=1.196, y_{g}=11.83, z_{g}=2$ and $\mathbf{R}=\mathbf{I}_{\mathbf{3}}$. At this pose we have $2 \mathrm{IK}$ solutions $\mathbf{X}_{\mathbf{1}}, \mathbf{X}_{\mathbf{2}}$, that have been calculated using the algorithm described in [21]. Starting from this pose we increase $x_{g}$ by a small increment $\epsilon$ so that we may use the Newton scheme to compute the IK solutions at the new pose using as initial guess $\mathbf{X}_{\mathbf{1}}$ and then $\mathbf{X}_{\mathbf{2}}$. The increment $\epsilon$ is chosen so that the Kantorovitch theorem ensures that that there is a single solution of the new IK system in the neighborhood of the initial guess and that this solution is guaranteed to be found by the Newton scheme [25]. We have implemented in the Maple interface of our ALIAS library a Newton scheme that allows one to compute the root of an arbitrary system with an arbitrary accuracy (we specify the desired number of digits $m$ and we guarantee that the $m$-th digit of the provided estimation of the solution does not differ from the corresponding digit of the exact root by more than \pm 1 ).

If the Kantorovitch conditions hold for the IK system, then the Newton scheme will converge and we have established the IK solutions for the new $x_{g}$ and we repeat the process. If the conditions do not hold, then we divide $\epsilon$ by 2 and repeat the process unless $\epsilon$ is lower than a fixed threshold (typically 1e-40). If the Newton scheme does not converge for this $\epsilon$, then we may suspect that we are close to a singularity but 1) we still have to prove that we are close to a singularity, 2) we have to determine exactly the location singular pose and 3) we have to determine the singularity type.

For proving that there is a singularity and for finding its location we will consider the 24 equations system $\mathcal{G}$ (equations 2, 3 and 4) where we let $x_{g}$ and the $\alpha$ be unknowns so that we have 25 unknowns. We also add as additional constraints $\left|\mathbf{J}_{\mathbf{I K}}\right|=0$ to construct a system $\mathcal{G}_{1}$ which is square. Note that $\mathbf{J}_{\mathbf{I K}}$ has been established by assuming that $x_{g}, \alpha$ where known. We may however remove this assumption and establish this matrix as a function of $F_{x}, F_{z}, L_{0}, x_{g}, \alpha$. Finding the root of $\mathcal{G}_{1}$ will provide the $x_{g}$ of the singularity. As we have already got a good approximation of this root we may use the Newton scheme to calculate the root. The problem is that the determinant of the $18 \times 18 \mathbf{J}_{\mathbf{I K}}$ cannot be computed in closed-form, while we require its derivatives 
with respect to the 25 unknowns. However this not a problem as one of the derivative of this determinant is the sum of the determinants of 18 matrices that are obtained by substituting in turn each row of the jabobian by the derivatives of the elements of this row with respect to the variable. Using this approach we have determined that the singularity was located at $x_{g}=1.1989510436242703461$ with $\left|\mathbf{J}_{\mathbf{I K}}\right|<1 e-200$, while the $|\mathbf{J}|=0.08538$, which show that we have indeed an IK full singularity and not a parallel robot singularity. Note that at the singularity we have the lowest $F_{x}$ equal to 1.1115666 . Figure 3 shows the determinant of $\mathbf{J}_{\mathbf{I K}}$ for the two IK branches obtained by following the branches starting from $\mathbf{X}_{\mathbf{1}}, \mathbf{X}_{\mathbf{2}}$ and the determinant of $\mathbf{J}$ which is clearly not 0 at the singularity. Figure 4 shows the value
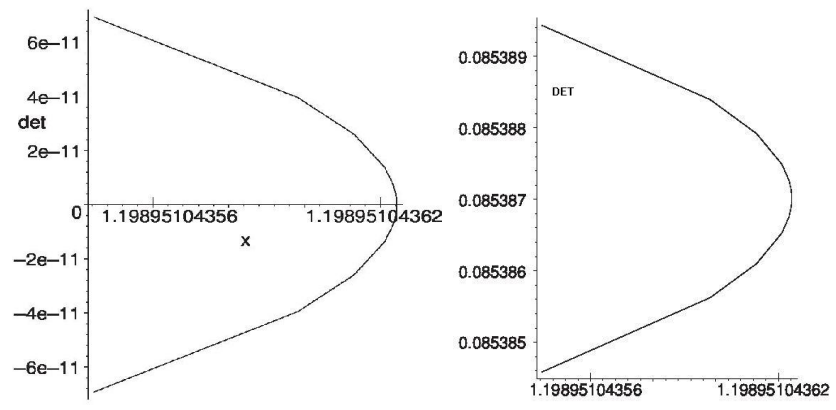

Fig. 3. On the left the determinant of $\mathbf{J}_{\mathbf{I K}}$ for the two IK branches that meet at the singularity. On the right the determinant of $\mathbf{J}$ which is not 0 at the singularity.

of $L_{0}^{1}$ for the two IK branches: we see clearly that these two branches meet at the singularity. This figure also shows a detail of the workspace when the cable length values are limited to 25 (obtained with the algorithm described in [26]): the workspace borders corresponding to the 2 different IK solutions are either due to the cable length limitation or is the singular curve (denoted sing) on the figure. This curve includes the singular pose we have obtained. Under this curve the IK has two distinct solutions, while the IK has no solution over the curve.
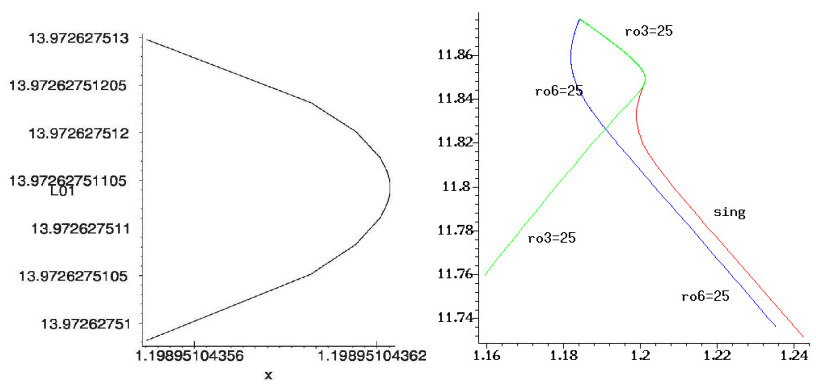

Fig. 4. The value of $L_{0}^{1}$ for the two IK branches which meet at the singularity. On the right a detail of the cross-section of the workspace.

\section{FORWARD KINEMATICS SINGULARITY}

As seen previously the FK may be written as a square system of $3 n+6$ equations $\mathbf{F}_{\mathbf{F K}}\left(\mathbf{X}, F_{x_{i}}, F_{z_{i}}, \alpha_{i}\right)=0$ or equivalently $\mathbf{F}_{\mathbf{F K}}\left(\mathbf{X}, \tau_{i}, \alpha_{i}, \beta_{i}\right)=0$. We already know that this system may have multiple solutions [27]. Using equation (6) the jacobian matrix $\mathbf{J}_{\mathbf{F K}}$ of this system may be written as:

$$
\mathbf{J}_{\mathbf{F K}}=\left(\begin{array}{cccc}
\mathrm{B}_{1} & \mathrm{C}_{1} & \mathrm{C}_{2} & \mathrm{C}_{3} \\
\mathrm{~B}_{2} & \mathbf{0}_{n \times 2 n} & & \mathrm{C}_{1}^{\alpha} \\
\mathbf{0}_{6 \times 6} & \mathrm{C}_{1}^{s} & \mathrm{C}_{2}^{s} & \mathrm{C}_{3}^{s}
\end{array}\right)
$$

We may classify the direct kinematics singularities in the same way than for the inverse kinematics with the Irvine singularity and FK parallel robot singularity. Indeed if we consider the matrix constituted of the last 6 rows of $\mathbf{J}_{\mathbf{F K}}$ as $\mathrm{C}_{3}^{\mathrm{S}}$ has rank at most 5 , then the rank of this matrix is at most the rank of $\mathbf{J}$. Consequently if $\mathbf{J}$ is singular then so will be $\mathbf{J}_{\mathbf{F K}}$. Now it remains to prove that full $F K$ singularity, at which 2 FK solution branches meet, may exist as, to the best of the author knowledge, such a singularity has never been exhibited. We will prove their existence on an example.

\section{A. Full FK singularity examples}

For this example we use the 8-cables CDPR COGIRO that has been developed by LIRMM and Tecnalia [28]. This robot is a suspended CDPR (i.e. there is no cable pulling the platform downward) with 8 cables, whose $A_{i}, B_{i}$ coordinates are given in table II. We have exhibited in [27] an example

\begin{tabular}{|c|c|c|c|c|c|c|c|c|}
\hline & $x_{A}$ & \multicolumn{2}{|c|}{$y_{A}$} & $z_{A}$ & $x_{A}$ & $y_{A}$ & \multicolumn{2}{|c|}{$z_{A}$} \\
\hline & -7.175 & \multicolumn{2}{|c|}{-5.244} & 5.462 & -7.316 & -5.1 & 5.47 & \\
\hline & -7.3 & \multicolumn{2}{|c|}{5.2} & 5.476 & -7.161 & 5.3 & 5.485 & \\
\hline & 7.182 & \multicolumn{2}{|c|}{5.3} & 5.488 & 7.323 & 5.2 & 5.499 & \\
\hline & 7.3 & -5 & & 5.489 & 7.161 & -5.27 & 5.497 & \\
\hline$x_{B}$ & \multicolumn{2}{|c|}{$y_{B}$} & \multicolumn{2}{|c|}{$z_{B}$} & $x_{B}$ & \multicolumn{2}{|c|}{$y_{B}$} & $z_{B}$ \\
\hline 0.5 & \multicolumn{2}{|c|}{-0.492} & \multicolumn{2}{|c|}{0} & -0.5 & \multicolumn{2}{|c|}{0.35} & 0.997 \\
\hline-0.5032 & \multicolumn{2}{|c|}{-0.2699} & \multicolumn{2}{|c|}{0} & -0.50321 & \multicolumn{2}{|c|}{0.49283} & 0 \\
\hline 0.4960 & \multicolumn{2}{|c|}{0.35562} & \multicolumn{2}{|c|}{0.99954} & 0.49964 & \multicolumn{2}{|c|}{-0.34028} & 0.99918 \\
\hline 0.5020 & \multicolumn{2}{|c|}{0.2749} & \multicolumn{2}{|c|}{-0.00062} & -0.50454 & \multicolumn{2}{|c|}{-0.34629} & 0.99752 \\
\hline
\end{tabular}

TABLE II

CoOrdinates of the $A_{i}, B_{i}$ POINTs (In METERs) of COGIRO

with $50 \mathrm{FK}$ solutions using the length $L_{0}$ that correspond to the IK solution for the pose $(1,0,2)$ with a rotation matrix equal to the identity. To investigate possible singularity we have decided to examine what will happen if we reduce the cable length $L_{0}^{1}$, that is initially 10.48214993 , while keeping constant the other lengths. We have considered each of the $50 \mathrm{FK}$ solutions and decreased the length $L_{0}^{1}$ incrementally using a continuation scheme to solve the FK at each step. Each step increment $\epsilon$ is determined using the Kantorovitch theorem to ensure that we stay on the same kinematic branch. For a given FK the process is stopped either if $L_{0}^{1}<0.1$ or if the Newton scheme does not converge even for $\epsilon=1 \mathrm{e}-40$.

As for the IK the non convergence of the Newton scheme only give a (good) indication of the closeness to a singularity and an estimation $\hat{\mathbf{X}}_{\mathbf{s}}$ of it location $\mathbf{X}_{\mathbf{s}}$. To refine this estimation of this location we proceed as for the IK. We consider an augmented system with $L_{0}^{1}$ as additional unknown and $\left|\mathbf{J}_{\mathbf{F K}}\right|=0$ as additional constraint and we then use the Newton scheme to solve the augmented system, using $\hat{\mathbf{X}}_{\mathbf{s}}$ 
as initial guess. To further confirm that in the close vicinity of $\hat{\mathbf{X}}_{\mathbf{s}}$ there is indeed a root of the augmented system, we may again use the Kantorovitch theorem. However using this theorem implies to be able to calculate an upper bound of the norm of the Hessian matrix when the unknowns have interval values. Although this is possible, we cannot explain this process in this paper for lack of space. However the results that will be presented in this section have been obtained using this certification process.

Table IV gives, for each of the $50 \mathrm{FK}$ solutions, the value of $L_{0}^{1}$ at which the process has stopped, the value of the determinant of $\mathbf{J}_{\mathbf{F K}}$ and of $\mathbf{J}$ at this pose. The continuation process has stopped because $L_{0}^{1}<0.1$ for the two solutions $(9,12)$, while for the 48 other solutions it stops because of the non convergence of the Newton scheme.

Among these 48 kinematic branches, 7 pairs ends up at the same platform pose: $3-16,10-15,17-18,24-50,28-46$, $37-41,38-45$ and therefore at these poses we have indeed a full FK singularity. For the remaining 34 singularities we notice that $|\mathbf{J}|$ is not 0 so that we don't have parallel robot singularity. A check of the jacobian of the IK system for these 34 poses shows that they all have full rank although the condition number of $\mathbf{J}_{\mathbf{I K}}$ may be quite large (see table III). As may be seen in this table for many of these 34 FK solutions we have cables that are almost horizontal with very large $F_{x}$. For example figure 5 shows the singularity 2 , while figure 6 shows the condition number of $\mathbf{J}_{\mathbf{I K}}$ as a function of $L_{0}^{1}$ for this singularity. In this configuration we have 4 extremely taught cables while the 4 others are sagging. As the IK is not singular for this 34 configurations

\begin{tabular}{|c|c|c|c|c|c|c|c|c|}
\hline sol & $\mathrm{c}$ & $F_{x}^{m}$ & sol & $\mathrm{c}$ & $F_{x}^{m}$ & sol & $\mathrm{c}$ & $F_{x}^{m}$ \\
\hline 1 & $1.51 \mathrm{e} 7$ & $5.73 \mathrm{e} 6$ & 2 & $4.62 \mathrm{e} 4$ & $1.00 \mathrm{e} 4$ & 3 & $6.74 \mathrm{e} 2$ & $1.44 \mathrm{e} 2$ \\
\hline 4 & $1.31 \mathrm{e} 7$ & $5.75 \mathrm{e} 6$ & 5 & $6.24 \mathrm{e} 4$ & $9.99 \mathrm{e} 3$ & 6 & $2.83 \mathrm{e} 6$ & $6.63 \mathrm{e} 5$ \\
\hline 7 & $9.31 \mathrm{e} 5$ & $3.79 \mathrm{e} 5$ & 8 & $1.11 \mathrm{e} 5$ & $3.02 \mathrm{e} 4$ & 10 & $4.51 \mathrm{e} 2$ & $1.16 \mathrm{e} 2$ \\
\hline 11 & $2.41 \mathrm{e} 3$ & $6.08 \mathrm{e} 2$ & 13 & $1.53 \mathrm{e} 6$ & $3.85 \mathrm{e} 5$ & 14 & $2.86 \mathrm{e} 6$ & $6.65 \mathrm{e} 5$ \\
\hline 15 & $4.51 \mathrm{e} 2$ & $1.16 \mathrm{e} 2$ & 16 & $6.74 \mathrm{e} 2$ & $1.44 \mathrm{e} 2$ & 17 & $7.48 \mathrm{e} 5$ & $1.14 \mathrm{e} 5$ \\
\hline 18 & $7.48 \mathrm{e} 5$ & $1.14 \mathrm{e} 5$ & 19 & $7.28 \mathrm{e} 4$ & $2.55 \mathrm{e} 4$ & 20 & $9.63 \mathrm{e} 4$ & $3.02 \mathrm{e} 4$ \\
\hline 21 & $2.51 \mathrm{e} 5$ & $2.56 \mathrm{e} 4$ & 22 & $1.18 \mathrm{e} 3$ & $2.41 \mathrm{e} 2$ & 23 & $2.85 \mathrm{e} 5$ & $3.72 \mathrm{e} 4$ \\
\hline 24 & $2.46 \mathrm{e} 3$ & $7.25 \mathrm{e} 2$ & 25 & $2.12 \mathrm{e} 5$ & $5.22 \mathrm{e} 4$ & 26 & $2.20 \mathrm{e} 5$ & $3.41 \mathrm{e} 4$ \\
\hline 27 & $9.09 \mathrm{e} 7$ & $1.48 \mathrm{e} 7$ & 28 & $4.86 \mathrm{e} 5$ & $3.30 \mathrm{e} 4$ & 29 & $7.75 \mathrm{e} 7$ & $1.51 \mathrm{e} 7$ \\
\hline 30 & $3.96 \mathrm{e} 6$ & $3.79 \mathrm{e} 5$ & 31 & $4.06 \mathrm{e} 5$ & $8.84 \mathrm{e} 4$ & 32 & $2.16 \mathrm{e} 5$ & $6.09 \mathrm{e} 4$ \\
\hline 33 & $3.87 \mathrm{e} 3$ & $9.17 \mathrm{e} 2$ & 34 & $4.16 \mathrm{e} 6$ & $7.81 \mathrm{e} 5$ & 35 & $4.02 \mathrm{e} 5$ & $9.22 \mathrm{e} 4$ \\
\hline 36 & $1.21 \mathrm{e} 3$ & $4.42 \mathrm{e} 2$ & 37 & $1.55 \mathrm{e} 6$ & $7.78 \mathrm{e} 5$ & 38 & $1.86 \mathrm{e} 3$ & $3.95 \mathrm{e} 2$ \\
\hline 39 & $1.63 \mathrm{e} 6$ & $3.85 \mathrm{e} 5$ & 40 & $3.83 \mathrm{e} 3$ & $7.79 \mathrm{e} 2$ & 41 & $1.55 \mathrm{e} 6$ & $7.78 \mathrm{e} 5$ \\
\hline 42 & $2.70 \mathrm{e} 6$ & $1.14 \mathrm{e} 5$ & 43 & $1.53 \mathrm{e} 3$ & $2.78 \mathrm{e} 2$ & 44 & $1.76 \mathrm{e} 3$ & $3.78 \mathrm{e} 2$ \\
\hline 45 & $1.86 \mathrm{e} 3$ & $3.95 \mathrm{e} 2$ & 46 & $4.86 \mathrm{e} 5$ & $3.30 \mathrm{e} 4$ & 47 & $3.85 \mathrm{e} 3$ & $4.84 \mathrm{e} 2$ \\
\hline 48 & $2.12 \mathrm{e} 3$ & $5.55 \mathrm{e} 2$ & 49 & $8.00 \mathrm{e} 2$ & $2.86 \mathrm{e} 2$ & 50 & $2.46 \mathrm{e} 3$ & $7.25 \mathrm{e} 2$ \\
\hline
\end{tabular}

TABLE III

THE CONDITION NUMBER $c$ OF $\mathbf{J}_{\text {IK }}$ AND THE MAXIMAL $F_{x}$ FOR ALL FK SINGULARITIES

we have not a FK combined singularity where both the IK and FK are singular. Consequently we may suspect that there is another FK branch that will lead to the same FK solution. To confirm this hypothesis we consider the FK system with solution $\hat{\mathbf{X}}_{\mathbf{s}}$ and perturb randomly all the set $L_{0}^{s}$ of $L_{0}$ at the singular configuration by a small amount (typically less than $0.01 \mathrm{~mm}$ ) to get a new set of lengths $L_{0}^{n}$.
We then use the Newton scheme with $\hat{\mathbf{X}}_{\mathbf{s}}$ as initial guess for solving the new FK system. If the scheme converge, then we have the starting point $\mathbf{X}_{\mathbf{n}}$ of a new FK branch. We then consider the FK problems $F K(\epsilon)$ where we set the $L_{0}$ to $L_{0}^{n}+\epsilon\left(L_{0}^{s}-L_{0}^{n}\right)$. Starting from $\epsilon=0(F K(0)$ has as solution $\mathbf{X}_{\mathbf{n}}$ ) we use a continuation scheme to solve $F K(\epsilon)$, increasing $\epsilon$ by incremental step (whose size is determined by the Kantorovitch theorem). For all 34 configurations $F K(\epsilon)$ becomes singular when $\epsilon$ comes close to 1 . This proves that all these configurations are singular because at least two FK solution branches meet at the singular pose.

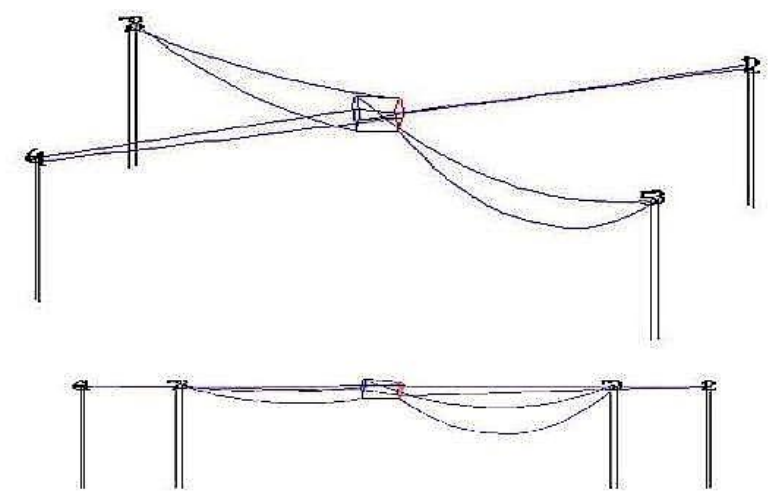

Fig. 5. The CDPR at the FK singular configuration 2: perspective and side view

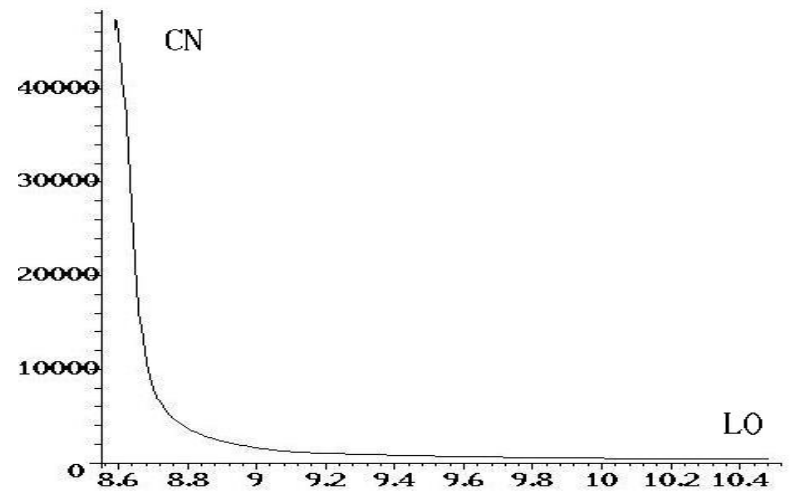

Fig. 6. The condition number $(\mathrm{CN})$ of $\mathbf{J}_{\mathbf{I K}}$ as a function of $L_{0}^{1}$ leading to the FK singular configuration 2 when $L_{0}^{1}$ decreases

\section{CONCLUSion}

We have shown in this paper that CDPR with sagging cables may have different singularities. A difference with classical parallel robots is that the IK and FK share the singularities of a classical parallel robot that is derived from the geometry of the CDPR (while for classical robot the IK is never singular except if the leg length is 0 ). Then we have IK and DK singularities that correspond to the intersection of two solution branches. For such IK singularity the pose will usually lie on the border of the workspace. Examples of all these singularities have been shown. After this preliminary classification still a lot of issues remains to be studied: 


\begin{tabular}{|c|l|l|l||l|l|l|l|}
\hline sol & $L_{0}^{1}$ & $\left|\mathbf{J}_{\mathbf{F K}}\right|$ & $|\mathbf{J}|$ & sol & $L_{0}^{1}$ & $\left|\mathbf{J}_{\mathbf{F K}}\right|$ & $|\mathbf{J}|$ \\
\hline 1 & 3.25 & $1.5 \mathrm{e}-103$ & $7.84 \mathrm{e}-07$ & 2 & 8.59 & $4.4 \mathrm{e}-96$ & $1.15 \mathrm{e}-03$ \\
\hline 3 & 9.60 & $-9.3 \mathrm{e}-91$ & $8.71 \mathrm{e}-03$ & 4 & 3.24 & $8.0 \mathrm{e}-103$ & $2.75 \mathrm{e}-06$ \\
\hline 5 & 8.59 & $1.1 \mathrm{e}-95$ & $5.32 \mathrm{e}-04$ & 6 & 8.89 & $7.3 \mathrm{e}-95$ & $7.53 \mathrm{e}-06$ \\
\hline 7 & 8.99 & $1.3 \mathrm{e}-94$ & $1.90 \mathrm{e}-06$ & 8 & 9.08 & $-5.3 \mathrm{e}-90$ & $2.62 \mathrm{e}-04$ \\
\hline 9 & - & - & - & 10 & 10.23 & $1.4 \mathrm{e}-88$ & $7.45 \mathrm{e}-02$ \\
\hline 11 & 9.52 & $1.7 \mathrm{e}-89$ & $1.10 \mathrm{e}-04$ & 12 & - & - & - \\
\hline 13 & 8.99 & $-2.5 \mathrm{e}-97$ & $3.53 \mathrm{e}-06$ & 14 & 8.88 & $-5.8 \mathrm{e}-96$ & $2.04 \mathrm{e}-05$ \\
\hline 15 & 10.23 & $1.4 \mathrm{e}-88$ & $7.45 \mathrm{e}-02$ & 16 & 9.60 & $-9.3 \mathrm{e}-91$ & $8.71 \mathrm{e}-03$ \\
\hline 17 & 8.50 & $-3.3 \mathrm{e}-105$ & $3.05 \mathrm{e}-07$ & 18 & 8.50 & $-3.3 \mathrm{e}-105$ & $3.05 \mathrm{e}-07$ \\
\hline 19 & 9.74 & $1.1 \mathrm{e}-88$ & $1.36 \mathrm{e}-04$ & 20 & 9.07 & $-2.2 \mathrm{e}-87$ & $1.36 \mathrm{e}-05$ \\
\hline 21 & 9.73 & $-9.4 \mathrm{e}-90$ & $1.01 \mathrm{e}-04$ & 22 & 8.98 & $1.6 \mathrm{e}-91$ & $1.53 \mathrm{e}-02$ \\
\hline 23 & 10.11 & $-5.0 \mathrm{e}-96$ & $3.16 \mathrm{e}-05$ & 24 & 9.35 & $1.1 \mathrm{e}-89$ & $2.82 \mathrm{e}-04$ \\
\hline 25 & 8.60 & $-9.4 \mathrm{e}-103$ & $1.42 \mathrm{e}-06$ & 26 & 10.11 & $4.6 \mathrm{e}-97$ & $1.01 \mathrm{e}-04$ \\
\hline 27 & 0.84 & $-3.0 \mathrm{e}-103$ & $5.18 \mathrm{e}-07$ & 28 & 8.69 & $2.5 \mathrm{e}-102$ & $5.22 \mathrm{e}-04$ \\
\hline 29 & 0.80 & $-1.5 \mathrm{e}-103$ & $1.87 \mathrm{e}-06$ & 30 & 8.99 & $1.2 \mathrm{e}-94$ & $1.66 \mathrm{e}-06$ \\
\hline 31 & 10.17 & $-4.4 \mathrm{e}-90$ & $7.98 \mathrm{e}-07$ & 32 & 8.92 & $1.2 \mathrm{e}-94$ & $1.06 \mathrm{e}-04$ \\
\hline 33 & 10.22 & $7.2 \mathrm{e}-89$ & $9.19 \mathrm{e}-05$ & 34 & 8.78 & $1.1 \mathrm{e}-99$ & $2.36 \mathrm{e}-05$ \\
\hline 35 & 10.16 & $-1.7 \mathrm{e}-90$ & $5.28 \mathrm{e}-06$ & 36 & 10.19 & $1.4 \mathrm{e}-90$ & $2.82 \mathrm{e}-02$ \\
\hline 37 & 8.79 & $-1.8 \mathrm{e}-99$ & $9.77 \mathrm{e}-07$ & 38 & 9.29 & $-3.0 \mathrm{e}-93$ & $2.62 \mathrm{e}-02$ \\
\hline 39 & 9.00 & $1.1 \mathrm{e}-95$ & $1.11 \mathrm{e}-04$ & 40 & 10.44 & $1.1 \mathrm{e}-88$ & $1.04 \mathrm{e}-03$ \\
\hline 41 & 8.79 & $-1.8 \mathrm{e}-99$ & $9.77 \mathrm{e}-07$ & 42 & 8.50 & $-4.0 \mathrm{e}-105$ & $7.60 \mathrm{e}-07$ \\
\hline 43 & 9.74 & $-8.8 \mathrm{e}-90$ & $2.25 \mathrm{e}-02$ & 44 & 10.35 & $2.8 \mathrm{e}-89$ & $3.29 \mathrm{e}-05$ \\
\hline 45 & 9.29 & $-3.0 \mathrm{e}-93$ & $2.62 \mathrm{e}-02$ & 46 & 8.69 & $2.5 \mathrm{e}-102$ & $5.22 \mathrm{e}-04$ \\
\hline 47 & 10.01 & $-2.7 \mathrm{e}-90$ & $2.72 \mathrm{e}-03$ & 48 & 9.67 & $-2.1 \mathrm{e}-90$ & $1.49 \mathrm{e}-03$ \\
\hline 49 & 10.29 & $-2.9 \mathrm{e}-90$ & $4.25 \mathrm{e}-03$ & 50 & 9.35 & $1.1 \mathrm{e}-89$ & $2.82 \mathrm{e}-04$ \\
\hline
\end{tabular}

TABLE IV

THE VALUE OF THE DETERMINANT OF THE JACOBIAN OF THE FK AND THE VALUE OF THE CLASSICAL DETERMINANT

- finding singularity curves in a plane: it seems to be difficult to determine a closed-form or parametric form for planar singularity curves while these curves are the border of workspace aspects and are therefore crucial. A strategy have been proposed to numerically approximate these curves [26] but it is computer intensive

- motion planning: find a strategy to move from one IK solution to another one without crossing a singularity

- workspace: find the largest singularity free workspace of a CDPR or check that a given workspace is singularityfree

- design: determine the influence of the CDPR geometry on the singularity

- investigate if there may be combined singularity where both IK and FK are singular, besides parallel robot singularity

\section{REFERENCES}

[1] R. Verhoeven, M. Hiller, and S. Tadoroko, "Workspace, stiffness, singularities and classification of tendon driven Stewart platforms," in ARK, Strobl, June 29- July 4, 1998, pp. 105-114.

[2] X. Diao, O. Ma, and Q. Lu, "Singularity analysis of planar cabledriven parallel robots," in IEEE Conference on Robotics, Automation and Mechatronics, Chengdu, September 2008, pp. 272-277.

[3] X. Diao, "Singularity analysis of fully-constrained cable-driven parallel robots with seven cables," in IEEE International Conference on Mechatronics and Automation (ICMA), Beijing, August 2015, pp. 1537-1541.

[4] S. Bandyopadhyay and A. Ghosal, "Analysis of configuration space singularities of closed-loop mechanisms and parallel manipulators,"
Mechanism and Machine Theory, vol. 39, no. 5, pp. 519-544, May 2004.

[5] J. Hubert and J.-P. Merlet, "Static of parallel manipulators and closeness to singularity," J. of Mechanisms and Robotics, vol. 1, no. 1, February 2009. [Online].

[6] J.-P. Merlet, "A formal-numerical approach for robust in-workspace singularity detection," IEEE Trans. on Robotics, vol. 23, no. 3, pp. 393-402, June 2007. [Online].

[7] A. Müller, "Higher order local analysis of singularity of parallel mechanisms," in ASME Design Engineering Technical Conference, Montréal, September 29- October 2, 2002.

[8] K. Kozak et al., "Static analysis of cable-driven manipulators with non-negligible cable mass," IEEE Trans. on Robotics, vol. 22, no. 3, pp. 425-433, June 2006.

[9] N. Riehl et al., "On the static workspace of large dimension cablesuspended robots with non negligible cable mass," in 34th Annual Mechanisms and Robotics Conference, Montréal, August, 15-18, 2010.

[10] D. Sridhar and R. Williams II, "Kinematics and statics including cable sag for large cable suspended robots," Global Journal of Researches in Engineering: H Robotics \& Nano-Tec, vol. 17, no. 1, 2017.

[11] M. Such et al., "An approach based on the catenary equation to deal with static analysis of three dimensional cable structures," Engineering structures, vol. 31, no. 9, pp. 2162-2170, 2009.

[12] M. Ahmadi-Kashani and A. Bell, "The analysis of cables subject to uniformly distributed loads," Eng. Struct., vol. 10, pp. 174-182, 1988.

[13] H. Hussein, M. Gouttefarde, and F. Pierrot, "Static modeling of sagging cables with flexural rigidity and shear forces," in $A R K$, Bologna, July, 1-5, 2018.

[14] S. Ghoreishi et al., "Analytical modeling of synthetic fiber rope. part II: a linear elastic model for 1+6 fibrous structure," Int. J. of Solids and Structures, pp. 2943-2966, 2007.

[15] J. Piao et al., "Open-loop position control of a polymer cabledriven parallel robot via a viscoelastic cable model for high payload workspaces," Advances in Mechanical Engineering, vol. 9, no. 12, 2017.

[16] W. Samuel et al., "Synthetic mooring rope for marine renewable energy applications," Renewable energy, vol. 83, pp. 1268-1278, November 2015.

[17] V. Schmidt, "Modeling techniques and reliable real-time implementation of kinematics for cable-driven parallel robots using polymer fiber cables," Ph.D. dissertation, Université Stuttgart, June, 20, 2016.

[18] P. Tempel, F. Trautweing, and A. Pott, "Experimental identification of stress-strain material models of UHMWPE fiber cables for improving cable tension control strategies," in ARK, Bologna, July, 1-5, 2018.

[19] H. M. Irvine, Cable Structures. MIT Press, 1981.

[20] N. Riehl et al., "On the determination of cable characteristics for large dimension cable-driven parallel mechanisms," in IEEE Int. Conf. on Robotics and Automation, Anchorage, May, 3-8, 2010, pp. 47094714.

[21] J.-P. Merlet, "A new generic approach for the inverse kinematics of cable-driven parallel robot with 6 deformable cables," in $A R K$, Grasse, June, 27-30, 2016. [Online].

[22] J. C. Polking, "A survey of removable singularities," in Seminar on Nonlinear Partial Differential Equations, S. S. Chern, Ed. New York, NY: Springer New York, 1984, pp. 261-292.

[23] J.-P. Merlet and C. Gosselin, Handbook of Robotics. Heidelberg: Springer, 2008, ch. Parallel Mechanisms and Robots, pp. 269-285.

[24] J.-P. Merlet and D. Daney, "A portable, modular parallel wire crane for rescue operations," in IEEE Int. Conf. on Robotics and Automation, Anchorage, May, 3-8, 2010, pp. 2834-2839. [Online].

[25] R. Tapia, "The Kantorovitch theorem for Newton's method," American Mathematic Monthly, vol. 78, no. 1.ea, pp. 389-392, 1971.

[26] J.-P. Merlet, "Computing cross-sections of the workspace of a cable-driven parallel robot with 6 sagging cables having limited lengths," in ARK, Bologna, July, 1-5, 2018. [Online].

[27] — "A generic numerical continuation scheme for solving the direct kinematics of cable-driven parallel robot with deformable cables," in IEEE Int. Conf. on Intelligent Robots and Systems (IROS), Daejeon, October, 9-14, 2016. [Online].

[28] M. Gouttefarde et al., "Simplified static analysis of large-dimension parallel cable-driven robots," in IEEE Int. Conf. on Robotics and Automation, Saint Paul, May, 14-18, 2012, pp. 2299-2305. 